\title{
Physical Activity, Mental Health, and Wellbeing among Older Adults in South and Southeast Asia: A Scoping Review
}

\author{
Shanti Kadariya, ${ }^{1}$ Rupesh Gautam ${ }^{(D)},{ }^{2}$ and Arja R. Aro ${ }^{1}$ \\ ${ }^{1}$ Unit for Health Promotion Research, University of Southern Denmark, Esbjerg, 6700, Denmark \\ ${ }^{2}$ School of Public Health and Social Work, Queensland University of Technology, Kelvin Grove 4059, Brisbane, Australia \\ Correspondence should be addressed to Rupesh Gautam; rupesgautam@gmail.com
}

Received 23 April 2019; Revised 9 July 2019; Accepted 25 July 2019; Published 17 November 2019

Academic Editor: H.-X. Wang

Copyright ( 2019 Shanti Kadariya et al. This is an open access article distributed under the Creative Commons Attribution License, which permits unrestricted use, distribution, and reproduction in any medium, provided the original work is properly cited.

Background. Physical activity is believed to enhance body functions and sense of wellbeing in general population. Objectives. This study aimed to explore physical activity measures; and the association between those measures, and mental wellbeing among older adults in South and Southeast Asia. Methods. A systematic search was made in CINHAL, EMBASE, PubMed, and PsycINFO. Articles published between 2008 and 2018 were selected with participants aged 60 years and above, living at home, community, supported housing, or residential care homes, with no diagnosed/limiting illness. Results. Five observational and four interventional studies on physical activity were analysed. Depression and sleep quality were the commonest outcome variables. Exercise frequency, regularity, and duration were found to positively impact mental wellbeing. Conclusion. Physical activity was generally found protecting against depression and improved sleep quality of older adults from South and Southeast Asia. Future studies should focus on more objective measures of physical activity.

\section{Introduction}

1.1. Background. Ageing is a chronic condition of human life cycle and is accompanied by conditions such as cardiovascular disease, hypertension, diabetes mellitus, cancer, and/or mental illness [1].

Ageing is a long-term result of accumulation of several kinds of molecular and cellular damage over time. This stage in the life cycle is marked by a gradual decline in physical and mental capacity, increased risk of disease and finally death. Ageing is not just biological but also has a social facet to it marked by retirement, relocation to new living arrangements as well as deaths of partners, friends, and relatives [2].

Globally, the population is ageing rapidly, and it is estimated that older people or elderly, defined as those aged 60 years and over, will constitute $22 \%$ of the total world population by 2025 [3]. Older people have limited regenerative activities but are more prone to the increase in age-related diseases and disabilities, with several social and financial implications to the individual themselves and to the society. Furthermore, mental health issues constitute a critical aspect of health problems in older adults. It is estimated that more than $20 \%$ of older adults aged $60+$ years suffer from mental and neurological disorders globally. Mental and neurological disorders account for $6.6 \%$ of the total disability (DALYs) for this age group [4].

Due to several reasons like lack of independence, frailty, illness, separation, isolation, and simply due to their age, among other reasons, older people are at disproportionately higher risk of suffering from mental health problems. What is further noteworthy for all age groups, but especially so for the older adults, is that physical health has an impact on mental health just like mental health has an impact on physical health [4]. Therefore, along with a continued focus on extending their life expectancy, it is also necessary to understand the strategies to maintain sound mental health and wellbeing in older adults [5].

Physical activity refers to any bodily movement produced by skeletal muscles that needs energy as an input. It includes all daily activities like playing, carrying out household chores, travelling, and activities during work and recreational pursuits [6]. 
Participation in light and moderate physical activities have been known to be effective on delaying the functional decline among older adults, thereby promoting healthy ageing in them [7]. Different studies have assessed the relationship between active participation in exercise and sports and elderly wellbeing. Many of those studies found a positive correlation between exercise frequency, wellbeing, and body function improvement among older people. As a result, there is a level of understanding that regular participation in exercise habits enhances body functions and improves their sense of wellbeing. Some other benefits found by these studies are improvement in body coordination, flexibility, better sleep quality, decrease in depression and anxiety; and improvement in overall psychological wellbeing [8-10].

Mental health services in Southeast Asian countries have been reported as inadequate, and suffering from issues like lack of proper funding, lack of health workers and advocacy groups, urban-concentration of service providers with poor quality, and unaffordable services [11]. As such, the provision of mental health services in these countries is understood to be limited. With such inadequacies in mental health service provision for the general population, it is hard to expect a specific service focus on the mental health of the elderly population.

The state of mental health service provision in South Asia is not very promising either. Factors like large scale natural disasters and conflicts within the region have fuelled the poor state of mental health in South Asian population. Depression, anxiety, and posttraumatic stress disorders have been found to be some of the commonly occurring mental conditions, particularly resulting from conflicts. Apart from conflicts, other factors like female sex, displacement, being widowed/ divorced, low income and/or education and food insecurity have been identified as some of the reasons responsible for poor mental health status in this region. Old age is another reason cited to be responsible for the current state of mental health in South Asia, which is beginning to experience an unprecedented increase in the proportion of elderly population. Furthermore, lack of financing, lack of prioritization of mental health, stigma associated with mental health disorders, inadequate specialist health workers, and lack of integration with primary health care have been identified as some of the systemic reasons that have contributed to poor mental health service provision in the region [12].

1.2. Rationale. In the pretext of lack of priority, inadequate service provision and lack of specific interventions targeting the old age groups, focus on primordial and primary preventive measures could prove helpful in countries with rising older adult populations and limited resources. Studies on effectiveness of physical activity on mental health and wellbeing of senior adults have, to a great extent, been conducted in western populations or within western contexts. Due to their different socioeconomic contexts and cultures of work, retirement, and leisure than the western countries, having an understanding of such association between physical activity, mental health, and wellbeing among older people living in South and Southeast Asia is important. Although in limited number and focused on some specific countries of the region, studies have been conducted in the area of physical activity, mental health, and wellbeing among senior adults in South and Southeast Asia. However, attempts have not yet been made to gather the scattered information in one study. Therefore, we aimed to conduct a scoping review of the studies published in this research domain in this geographic area to generate an aggregated knowledge of all these studies and also to inform future studies.

1.3. Objectives. In order to map the literature, identify key concepts already explored and the existing gap in knowledge from South and Southeast Asia on physical activity and its influence on mental health and wellbeing among senior adults from the region, this study was conducted as a scoping review. Through a narrative overview, this study aimed to meet the following major objectives:

(i) Gather information on the types of physical activities and/or exercise measures studied in South and Southeast Asia in their relationship with mental health and wellbeing among older adults.

(ii) Understand the association between physical activity and/or exercise practices, mental health, and wellbeing among older adults in South and Southeast Asia.

(iii) Understand the effect of physical activity and/or exercise interventions on mental health and wellbeing among senior adults in South and Southeast Asia.

\section{Materials and Methods}

This study followed the 5 stages of York framework outlined by Arksey and O'Malley, [13] and PRISMA Extension for Scoping Reviews (PRISMA-ScR) [14].

2.1. Identifying the Research Questions. We decided on the research questions as our guide for article search. The questions helped us identify the general characteristics of the study in terms of person, place, and time; different physical activities measured in everyday lives as well as those introduced as interventions, different forms of mental health outcome variables chosen by studies and the ways the results were presented. Our questions were as follows:

(i) What is known about physical activity and/or exercise, mental health, and wellbeing among older adults in South and Southeast Asia?

(ii) What different forms of physical activity and/or exercise practices have been studied with an aim to understanding their influence on mental health and wellbeing of older adults in South and Southeast Asia?

(iii) What is known about the association between those different forms of physical activity and/or exercise practices (observations as well as interventions), mental health, and wellbeing among senior adults in South and Southeast Asia? 
2.2. Identifying Studies Relevant to the Research Questions. In order to decide on the studies to be included in the review, we defined some inclusion and exclusion criteria before beginning our literature search. Once we had developed our search criteria, we decided on the databases to search to look for the articles.

2.3. Inclusion Criteria. Studies with participants aged 60 years and over; living at home, in the community, in supported housing or in residential care homes; with no diagnosed illness/limiting illness; and who were able to walk without assistance were selected. Only those studies which assessed mental health and wellbeing of older adults in relation to physical activity were included. We limited our search to only those studies that included older adults with no diagnosed illness or limiting illness because we wanted to understand how physical activity influences supposedly healthy older adults' mental health and wellbeing. While exploring this research question in older adults with one or multiple morbidities is equally important, it is outside the scope of this study. Mental wellbeing, for the purpose of this study was defined to include better sleep, reduced depression and anxiety, and/or similar other pyschological outcomes. We only reviewed studies conducted in the last 10 years in order to incorporate relatively recent findings. Therefore, observational or experimental studies conducted between 2008 and 2018 in South Asian and Southeast Asian countries targeted at older adults aged 60 years and above [according to the classification of Asian Development Bank (ADB) [15] and South Asian Association for Regional Cooperation (SAARC)] [16] were included.

2.4. Exclusion Criteria. The studies were excluded if they considered older adults undergoing (a) treatment for a clinically diagnosed physical or mental illness, (b) assessment(s) for long-term continuing care, (c) and community interventions to improve physical and social environment not directly trageted at people aged 60 years and above.

2.5. Information Sources. The searches for articles were made in four databases: CINHAL, EMBASE, PubMed, and PsycINFO. The search targeted only English language studies with full access published between 2008 and 2018 .

2.6. Key Terms and Article Search. Key terms used for the exposure variables were Physical activity OR Exercise. Key terms used for mental health and wellbeing were Mental health, Mental Disorder, Depression, Anxiety, Wellbeing, Wellbeing, Well being, Psychological health, Self-esteem, Selfesteem, Self-perception, and self perception. The terms used to locate older participants were Aging, Ageing, Aged, Frail, Elderly, Senior citizens, Old age, Senior adults, Adult, Older, Elderly, Elder and Geriatric. Key terms used for the countries were Southeast Asia, South East Asia, and South Asia. Searches for the countries were also made including the individual names of all the countries in these two regions (see Supplementary Material 1 for full electronic search strategy in PubMed).

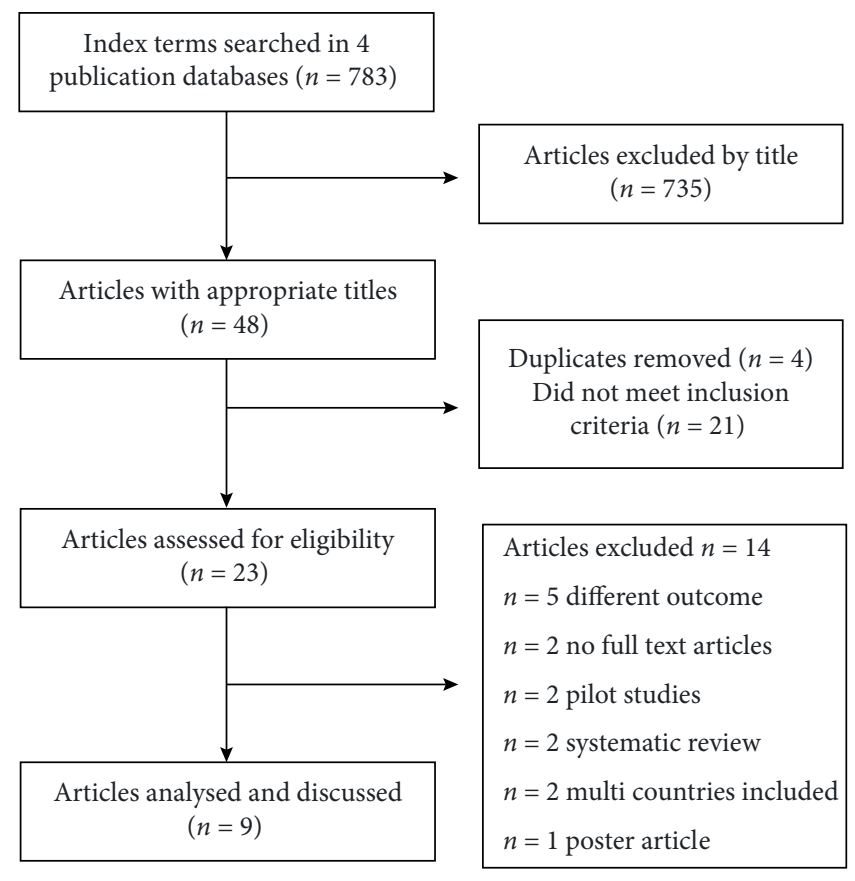

FIGURE 1: Flow chart showing the selection of studies included in the review.

Selection of sources of evidence: The article selection process is illustrated in Figure 1. At the first stage of selection, SK and RG considered the abstracts for inclusion and exclusion. Only peer-reviewed empirical articles were selected. All articles that met the inclusion criteria were then considered relevant and retrieved for further review. Articles were also identified from the reference lists of articles found during the search process. In the full review, those articles with outcome variables of older adults' mental health and wellbeing measured in relation to physical activity were considered. The articles failing to meet these criteria were rejected. All duplicate articles were removed from the list, and so were the pilot studies, and the studies to which the authors could not get full access. Only those articles that were written in English were selected.

2.7. Data Charting Process. We extracted information about each study's design, country of origin, data sources, population characteristics (sample size, age range, population type), intervention details, key findings, and wellbeing measures. We identified the variables that were used to assess mental health and wellbeing of older adults, and also the measures of physical activity and exercises (observations and interventions). Thereafter, we isolated the results for analyses. After that, analysis was conducted by the research team through review and re-review of the findings of the included articles.

2.8. Synthesis of Results. The information from the selected studies were organized in tables. The final version of the table has been presented in the results section. We have discussed, compared, and contrasted the findings of different studies in the results section following the table. 
TABLE 1: Overview of the studies included in the review.

\begin{tabular}{lccc}
\hline Characteristics & Number of studies $(n=9)$ & Characteristics & Number of studies $(n=9)$ \\
\hline Country & 7 & Study design & 3 \\
Taiwan & 1 & Cross-sectional & 1 \\
Pakistan & 1 & Quasi experimental & 2 \\
Thailand & & Longitudinal & 1 \\
& & Randomized control trail & 2 \\
Data type & & Cluster randomized control trail & 6 \\
Primary & 8 & Sample size & 1 \\
Secondary & 1 & $<500$ & 2 \\
Study setting & & $500-1000$ & $>1000$ \\
Community based & & & \\
Facility based & 8 & & \\
\hline
\end{tabular}

\section{Results}

3.1. Study Characteristics. Of the 9 articles that were selected for the final analyses, 7 were conducted in Taiwan and 1 each in Pakistan and Thailand as shown in Table 1. The settings of these studies were mostly community $(n=8)$ and only one was facility-based. The study designs adopted in these studies were cross sectional $(n=3)$, quasi experimental $(n=3)$, longitudinal $(n=1)$, and randomized controlled trial $(n=2)$. So, for the purpose of analyses, the studies were divided into two broad categories: the studies involving interventions $(n=4)$ and the studies with no intervention involved $(n=5)$. The sample size ranged between 50 and 1160 .

\subsection{Measurement of Physical Activity as Exposure Variable} in the Observational Studies. All five observational studies included in the review involved measuring physical activity using subjective measures (interview or self-reporting questionnaires). Three of the noninterventional studies were cross-sectional $[1,8,17]$, while the remaining two were prospective, longitudinal follow-up studies [5, 18]. All five of these studies involved older adults that were community dwelling, i.e., they were not selected from residential care facilities (Table 2).

To quantify physical activity, the respondents were asked about the kinds of activities they were involved in, the total time they were involved in those activities $[1,5,8,17,18]$ and the intensity of the activities $[1,18]$. In studies where intensity was not measured, the physical activity (PA) involvement was measured in terms of weekly frequency (hours/week). However, one study measured both intensity and weekly frequency [4]. Two studies measured physical activity in terms of regularity defined over the course of 6 months $[1,5]$. The defining criteria for regularity differed in these two studies in terms of how long each session lasted. The first of these two required only 10 minutes per session [1], while the second required every session to be at least 20-minute long [5].

The types of physical activity assessed included running, mountain climbing, swimming, walking up the stairs, aerobic exercise, fast and normal-speed bicycle riding, jumping ropes, carrying heavy things or babies, shovelling dirt, dancing, yard/ house work, playing tennis, playing baseball, and playing table tennis. Likewise, activities also included things done at employment/work or leisure like walking or exercising, shopping, meeting friends, carrying weights of at least $5 \mathrm{~kg}$ per week, time spent standing or sitting, and walking during work.

\subsection{Measurement of Physical Activity as Exposure Variable in} the Interventional Studies. As shown in Table 3, two of the four interventional studies adopted a quasi-experimental design and the remaining two studies were cluster-randomized trial and randomized controlled trial.

The interventions adopted for these studies were-the elastic band programme, the silver yoga exercise programme, Tai Chi, and Baduanjin exercise.

The longest intervention lasted for 6 months and the study data were collected at baseline, 3 months, and 6 months in this longest-lasting study out of the four [16]. Other studies were run for 3.5 months [19], 3 months [20], and 6 months [21], respectively. None of the control groups received any intervention and were followed up for their routine activity involvement. Two of these studies involved senior adults visiting activity centers [21,22], and one each involved senior adults living at residential care facility [19] and home [20].

\subsection{Variables Used to Quantify Mental Health in the} Observational Studies. Four out of five observational studies had depression as their mental health outcome variable [1, 5, $17,18]$. One of those studies however, had mental status as one more outcome variable in addition to depression [5]. The remaining observational study had wellbeing as its outcome variable; however, this variable also comprised depression as one of its six dimensions. Other dimensions included were anxiety, positive wellbeing, self-control, vitality, and general health [8].

Likewise, the Chinese version of the 10 -item Center for Epidemiological Studies-Depression Scale (CES-D) $[1,18]$ and the Geriatric Depression Scale $[5,17]$ were the two commonest 


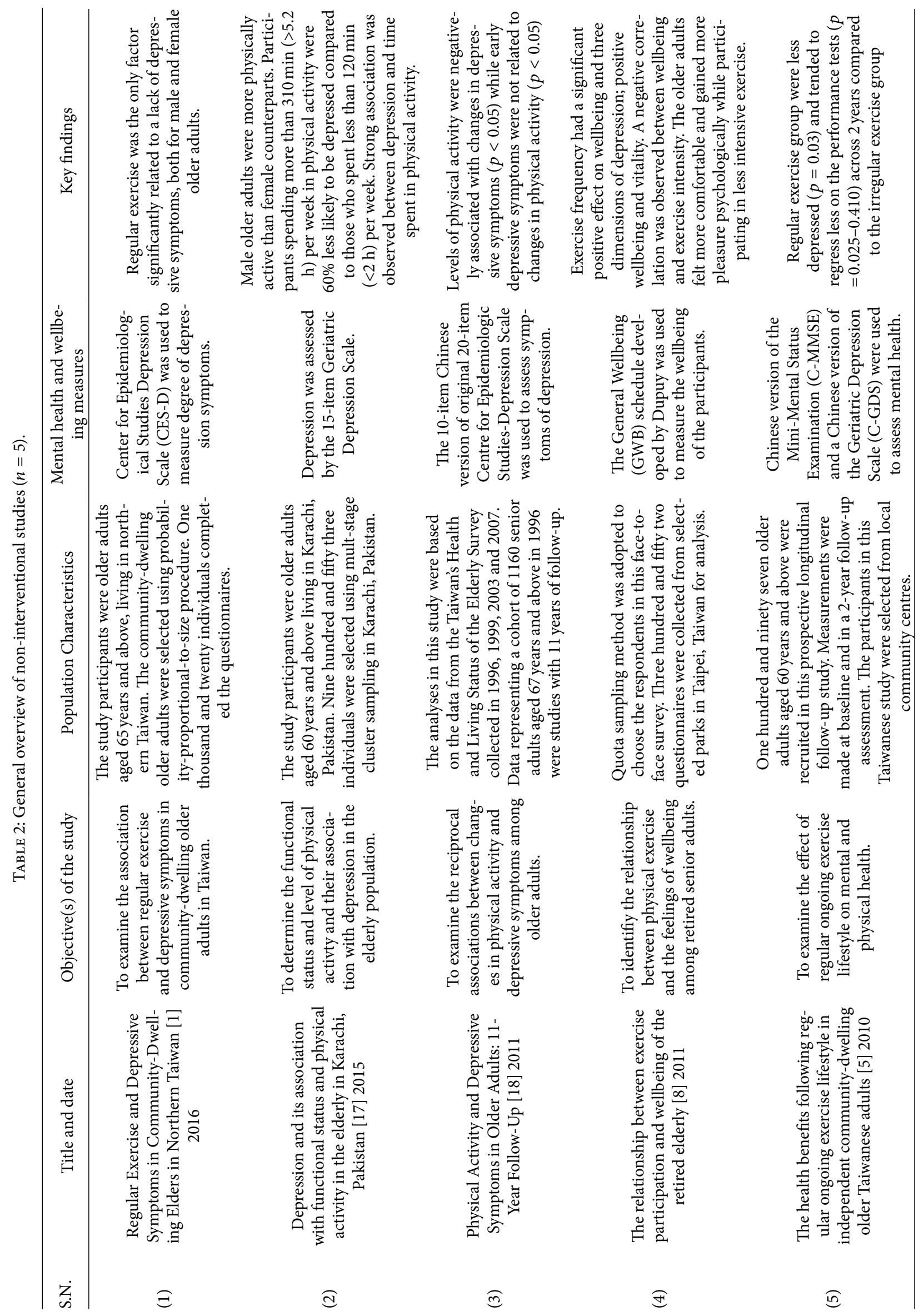




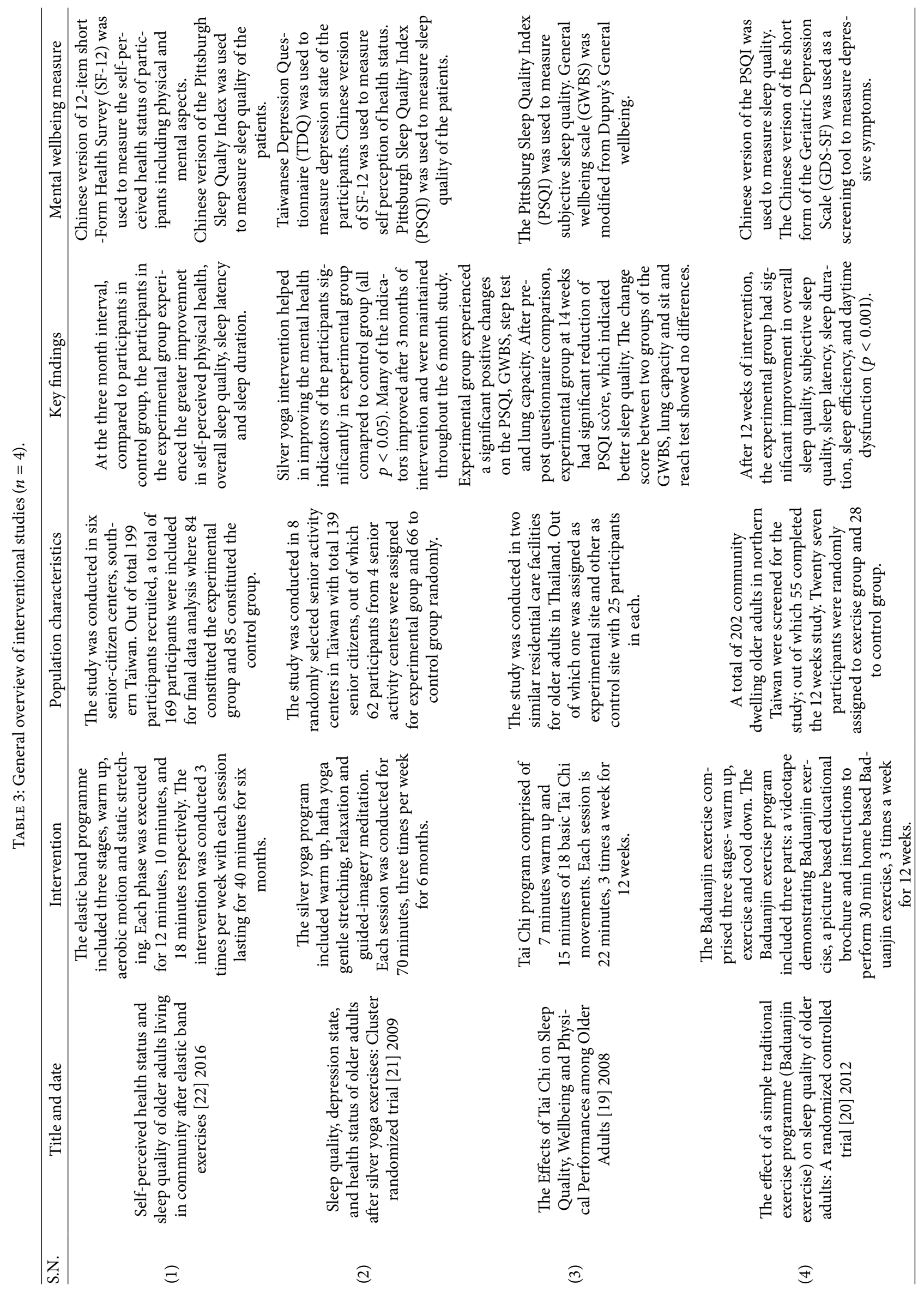


tools used for depression assessment in non-interventional studies. General wellbeing schedule was used by a study that employed wellbeing as its primary outcome variable [8].

\subsection{Variables Used to Quantify Mental Health in the} Interventional Studies. Three interventional studies used sleep quality as their outcome variable and it was measured by using the Chinese version of Pittsburgh Sleep Quality Index (PSQI) [19, 20, 22]. Likewise, in other studies, depression was assessed using the Taiwanese Depression Questionnaire [21], mental health perception was assessed using the mental health component of the Chinese version of SF-12 Health Survey [21], and mental component summary (MCS) derived from the Chinese version of the 12-item Short-form Health Survey (SF-12) was used to quantify self-perceived mental health status [22]. Just like in the study by Lee and Hung, wellbeing of senior adults was also measured using The General Wellbeing Scale (GWBS) in the study by Taboonpong, et al. $[8,20]$.

\subsection{Physical Activity Measurement Results in the Observational}

Studies. The non-interventional studies employed different criteria to determine physical activity among the participants involved. The study by Chang et al. considered each bout of 10 min for any activity to count as exercise while the criterion for the study from Pakistan was 20 minutes of activity every day to count as physical activity $[1,17]$. In addition to it, the Pakistani study also let the respondents rate their own intensity based on the levels of activity [17].

Walking was the commonest form of physical activity ranging from $43.8 \%$ in the Taiwanese adults [5] to (63.4\%) in the study among retired elders [8]. Other forms of exercises that were common were Chinese martial arts, swimming, dancing, bicycling, hiking, stretching, tennis, badminton, jogging, and golf $[5,8]$.

The studies that made comparison across genders found that men were more frequently active $[8,17]$, spent more time than women on average per week being physically active and exhibited low levels of no activity $[5,17]$. Likewise, men were also more likely to have more minutes spent in light activity and vigorous activity $(30.8 \%, 5.4 \%)$ than women $(25.1 \%, 5.1 \%)$ as demonstrated by the study from Pakistan [17]. In terms of diversity of activities, men tended to be more engaged in walking and hiking while women did more stretching in comparison. Likewise, women perceived hiking and yoga as too intense exercises, whereas men considered aerobics as intense as demonstrated by the study by Wang et al in Taiwan [5].

\subsection{Mental Health Outcomes with Respect to Physical Activity} in the Observational Studies. Those who were regularly physically active had lower odds of having depressive symptoms $(\mathrm{OR}=3.54$, CI: 1.76-7.12) according to the study in Northern Taiwan [1]. Wang et al. [5] also found that those who were irregular in their exercise were found to have higher depression $(p<0.05)$ compared to those who were regular. Mental status of the regulars and irregulars were also different, with those being regular in exercise more likely to have better mental health status over time $(p<0.05)$. Another Taiwanese study by Lee and Hung also found the exercise frequency to positively impact wellbeing in terms of depression, vitality and positive wellbeing among senior adults [8]. Similarly, the study from Pakistan found that a protection factor of $21.6 \%$ against depression could be attributed to spending more than $7 \mathrm{~h}$ every week in physical activity alone [17]. Final analyses also demonstrated that higher initial physical activity at baseline was associated with a slower increase in depressive symptoms, among those participants who had not already become depressed at baseline, thus implying future protection [18].

Some findings were, however, more specific in terms of duration and intensity of physical activity and suggested that the mental health-physical activity association might not always be straightforward. For example, Bhamani et al. in the study from Pakistan, pointed out that for the individuals to experience any benefit of being physically active against depression, the senior adults had to be active for more than 5.2 hours in a week. Therefore, those who only spent between 120 and 310 min per week in physical activity were not offered significant protection against depression (Adjusted $\mathrm{OR}=0.8$, 95\% CI $=0.4-1.2$ ) [17]. Additionally, according to the study by Chang et al., duration, frequency, and intensity of physical activity had no impact on the respondent's depressive symptoms [1]. Moreover, exercise intensity was even found to have a negative impact on individual wellbeing according to the study by Lee and Hung suggesting that too intense physical activity might do more harm than good to senior adults' mental health [8].

\subsection{Mental Health Outcomes with respect to Physical Activity in the Interventional Studies}

3.8.1. Sleep Quality. Senior adults in the intervention and control groups did not differ significantly in terms of their sleep quality at baseline in any of the three studies that measured sleep quality with respect to physical activity. However, end line comparisons showed that overall sleep quality improved in interventional groups but not in control groups in all three studies.

The study from Thailand did not present the results in terms of subscales of PSQI [20]. However, subscale comparisons between the study with elastic band exercise intervention and the study with the traditional Baduanjin exercise intervention showed mixed results even though the overall analyses showed better sleep quality in intervention groups than in control groups $[19,21]$.

Both sleep latency and sleep duration improved in the experimental groups compared to the control groups in the studies with elastic band exercise intervention and Baduanjin exercise intervention $[19,21]$. Sleep disturbances, daytime dysfunction, and use of sleep medications were not different between interventional and non-interventional groups in the study by Chan and Chen [19]. However, in the study by Chen et al., daytime dysfunction score was significantly worse in the control group as compared with the intervention group [21]. While subjective sleep quality showed significant improvement over the intervention period of 12 weeks in the study by Chen et al. [21], the study by Chan and Chen found that subjective sleep quality of experimental group improved at 3 months compared to the control group [19]; however, this was not maintained at 6 months. The study that used Tai Chi as intervention among older adults showed 
that the proportion of those with overall better sleep increased postintervention compared to preintervention from $20 \%$ to $32 \%$ [20]. However, the mean postintervention PSQI in the intervention group was still above the cut-off score of five suggesting overall dismal sleep quality. Noteworthy was also a finding in the study by Chen et al., that the sleep quality in intervention group declined gradually with time, but that for the control group remained unchanged [21].

To summarize, being involved in various forms of physical activity was found to positively contribute to overall better sleep quality among the senior adults as demonstrated by all three studies. However, there was no consistency in the findings for individual subscales.

3.8.2. Depression. The cluster randomized trial that used Silver Yoga exercise as intervention assessed the impact of the intervention on depression. Periodic analyses showed that the intervention group was better than control group in terms of depression state $(F=19.14, p=.000)$ after 3 months into the intervention period. Even after six months of intervention, the experimental group was better off than the control group $(p<0.05)$. Therefore, the overall analyses demonstrated that overall depression decreased $(F=10.92, p=.000)$ in the intervention group. These findings were in line with the observational studies, which also showed better mental health outcomes, measured in terms of decrease in depressive symptoms, among those who were more physically active than those who were not $[1,5,17,18]$.

\subsubsection{Other Outcomes (Mental Health Perception, Mental} Component Summary, and Wellbeing). The other two mental health outcomes showed mixed results. The study using Elastic band exercise intervention and the study using Silver Yoga intervention, with mental health perception as the outcome variable showed non-significant difference, and mental component summary showed no difference between the intervention and control groups at baseline $[19,22]$. Three months into the intervention period, the intervention group was better off than the control group $(F=5.59, p=0.020)$ in terms of mental health perception (MHP) [22]. Following six months of intervention, the experimental group was still better off than the control group $(p<0.05)[22]$.

On the contrary, mental component summary had neither any difference in mean scores between the intervention and control groups between baseline and three-month interval (95\% $\mathrm{CI}=-0.73$ to $5.05, p=0.143$ ) nor between the baseline and the six-month interval (95\% CI $=-0.91$ to $4.83, p=0.181)$ as seen in the study by Chan and Chen [19]. Furthermore, the MCS score declined over time in the intervention as well as in the control group. However, the decline was more pronounced in the control group than in the intervention group [19].

As shown by the study employing Tai Chi as the physical activity intervention, the measure of wellbeing increased significantly in experimental group but not in the control group after the intervention. However, the difference in change between the two groups was not statistically significant. Therefore, it was concluded that wellbeing was not significantly improved by the intervention [20].

\section{Discussion}

Alongside understanding the demographic characteristics of senior adults involved in physical activity, and the types of physical activity performed by them in selected countries of South Asia and South East Asia, this study was undertaken also to understand the effects of physical activity on mental health and wellbeing of older senior adults in those countries. The studies that met the inclusion criteria therefore were included in the final analyses were from 3 countries of Taiwan (7), Pakistan (1), and Thailand (1). The mental health outcomes that were assessed in these studies were depression [ 1 , $5,17,18,22]$, sleep quality [19-22], wellbeing $[8,20]$, and other measures like self-perceived mental status $[5,22]$ and mental component summary [19].

4.1. Demographic Differences in Physical Activity. It was found that most study participants in these studies fell inside the age bracket of 60 to 75 years. Most of these studies ( 7 out of 9 were from Taiwan) also demonstrated that these older people were physically active. This observation has also been made in the past in the context of Taiwan [23]. Older men were found to be more frequently active [18], spent more time being involved in a physical activity and tended to be more regular in their physical activity routine [5] compared to older women. This could also be a reflection of a cultural aspect of life in Pakistan [17] and Taiwan $[5,18]$ where women tend to stay indoors and take care of the housework while men tend to spend more time outdoors. However, when housework was also considered in daily physical activity as in the case of the study from Northern Taiwan, more women than men were found to be engaged in moderately vigorous physical activity [1]. This could also be suggestive of a higher engagement of women in indoor household activities than men. This also suggests that if housework is included in these subjective physical activity measures, women will probably not be seen as lagging behind men in terms of frequency, time spent as well as regularity of physical activity.

Coincidentally, three of the four interventional studies that were included in the review $[19,21,22]$ had higher number of women participants than men. It could also be suggestive of higher affinity among women to lighter physical activity than men. These studies included elastic band exercise, silver yoga, and Baduanjin exercise, which were all moderate to light physical exercises. Likewise, as seen in the study by Lee and Hung, older women found hiking and yoga to be very intense, while older men tended to perceive only aerobics as an intense activity. Along the same line, older men practiced walking and hiking more often while older women were more likely to be engaged in stretching [8].

4.2. Types of Physical Activity. All of the activities amounting to exercise or physical activity in this study were recorded subjectively. In the observational studies, depending on the operational definition of what comprised physical activity, participants were asked what activities they were engaged in. Walking for pleasure or exercise was the commonest form of light physical activity $[1,5,8,17,18,22]$. Other activities included lifting light weights up to $5 \mathrm{~kg}$, going to meet friends 
or relatives, gardening, going to purchase personal items [17], any kind of leisure time physical activity [18], hiking, jogging, stretching, aerobics; playing tennis, badminton or golf, and martial arts [8]. Likewise, participants were also found mentioning activities of independent daily living like dressing, eating, using toilet, bathing, and grooming and instrumental activities like using the telephone, light to heavy housework, preparing meals, shopping and outdoor transportation [5]. Likewise, ballroom or folk dancing, Tai Chi, qigong, and morning calisthenics were also some other activities mentioned by the study participants [22]. The interventional studies, on the other hand, introduced activities like elastic band exercise [19], Tai Chi [20], Baduanjin exercise [21], and silver yoga exercise [22].

4.3. Physical Activity and Depression. Duration, frequency, and regularity of physical activity were significantly associated with lowered levels of depression as demonstrated by studies by Bhamani et al., Ku et al., and Wang et al. [5, 17, 18]. In Pakistan, duration of physical activity was significantly associated with depression: participants who spent more than 310 min per week in physical activity, had $60 \%$ less likelihood to become depressed compared to the participants spending less than $120 \mathrm{~min}$ per week for physical activity [17]. In the 11-year follow-up study conducted in Taiwan, it was seen that the number of sessions of physical activity per week earlier in the study period of 11 years was predictive of reduced risk of depressive symptoms in the future [18]. Likewise, in another Taiwanese study, regular exercisers exhibited significant improvement in depression status, but the irregular exercisers showed regressed depression scores, emphasizing the importance of regular physical activity [5].

Another study from Northern Taiwan also found exercise regularity to be significantly associated with lower levels of depression. However, the study yielded a contradictory finding compared to other studies in terms of duration and frequency of physical activity. The study found that duration and frequency of physical activity had no influence upon depressive symptoms and neither had exercise intensity [1].

There was only one experimental study included in this review, which had depression as the mental health outcome. The Taiwanese study concluded that in a group of older adults who were not statistically different at baseline, the state of depression decreased in the experimental group who was exposed to silver yoga exercise, and it increased in the control group [22].

The positive effect of physical activity on depressive outcomes in senior adults has also been observed in studies across other regions of the world. For example: A 3-year follow-up study among senior adults between 65-75 years in the New York City found that non-disabled senior adults who were athletic, involved in walking or domestic gardening had lower odds of depression [24]. Similarly, intensity of baseline physical exercise was found to be one of the factors influencing future depressive symptoms among senior adults in Finland [25]. Likewise, a study among senior adults in Nottingham, UK also found a modest increase in the risk of depression among those who had lower levels of outdoor or leisure physical activity [26]. Similar was the finding of another study involving women aged 60 years and above in Northern New Jersey, i.e., the higher the levels of physical activity, the lower were the self-reported depressive symptoms. Likewise, in the same study, involvement in a line dancing intervention also led to significantly lower depression among the same group of women [27].

4.4. Physical Activity and Sleep Quality. The studies that assessed sleep quality as an outcome measure were all interventional in nature [19-22]. All of these studies unanimously found that sleep quality of the older adults who were in the experimental groups not only improved compared to the baseline but also showed significant positive difference with the sleep quality of older adults in the control groups. It was also found that the interventions started having positive impact on sleep quality within a short interval after the start of the intervention, within 4 weeks in the case of study by Chen et al. and within 3 months in the case of study by Chan and Chen. Those positive changes were found to continue throughout the remaining period of the interventions in both of these studies involving Elastic band exercise and Baduanjin exercise, respectively $[19,21]$.

Similar findings of better sleep quality among physically active senior adults have been found by studies conducted elsewhere. A systematic review and meta-analysis found that exercise can positively impact sleep quality in older adults [28]. Another meta-analysis found that moderate intensity aerobic exercise or high intensity resistance exercise between 10 and 16 weeks led to moderate beneficial effect on sleep quality as demonstrated by a reduced global Pittsburgh Sleep Quality Index score. Other subdomains of sleep like subjective sleep quality, sleep latency and sleep medication usage were also positively impacted by exercise training [29]. Likewise, another study from Chicago, USA also found that senior adults exposed to 16 weeks of aerobic physical activity and sleep hygiene education had better sleep quality compared to the controls. They also performed better in terms of sleep latency, sleep duration, daytime dysfunction, and sleep efficiency [30]. Another study from China among community-dwelling Chinese older adults found that regardless of factors like age, sex, education, family income, number of children, drinking and sleep hygiene, those who had a greater level of physical activity in comparison, were found to have a better quality of sleep outcome compared to their counterparts [31].

\subsection{Physical Activity, Wellbeing and Other Mental Health} Measures. Another outcome variable employed by two of the reviewed studies was wellbeing. It was used by one observational study by Lee and Hung and one interventional study by Taboonpong et al. The first study found that exercise frequency was positively associated with wellbeing. More frequent bouts of physical activity contributed to better general wellbeing in older adults. As explained above in the section of depression, physical activity frequency and depression had also shown a similar pattern: the higher the frequency, the lower the levels of depression $[8,20]$.

However, the same study found that high intensity exercise had a negative impact on the older adults' sense of wellbeing. Therefore, it implied that self-assessed low-to-moderate 
intensity exercise could be good for the psychological wellbeing of older adults when done frequently, but the outcome started shifting to the negative when physical activities got more intense. This finding is intuitive in the sense that older adults are engaged in physical activity in general not to demonstrate their endurance for high intensity physical activity but instead to keep themselves fit for their age and to reinforce their sense of wellbeing. This finding was also supported by similar findings from a meta-analysis where it was seen that longer exercise duration, which is naturally more tiring compared to exercise involving smaller shorter bouts, was less beneficial for the well-being of senior adults [32].

The experimental study involving Tai Chi intervention also found that general wellbeing had significant positive changes in the experimental group compared to the control group. However, even though there was a significant increase in wellbeing in the experimental group compared to the baseline, the difference in the wellbeing measure between the experimental and control group was not statistically significant [20].

The positive impact of physical activity on mental wellbeing as seen in the aforementioned studies was also demonstrated by previous studies. A meta-analysis of 13 studies reported that mental wellbeing in later life could be achieved through physical activity and exercise [33]. Another meta-analysis of 36 studies found that the mean-change effect size in mental wellbeing for physically active senior adults was almost 3 times that for those senior adults who were not physically active. Moderate intensity activity was found to have the most benefit in terms of mental wellbeing [32].

Self-perceived mental health status and mental component summary were also assessed in relation to physical activity in 3 studies [5, 19, 22]. Mental health status either improved or showed less deterioration among regular exercisers compared to irregular exercisers as demonstrated by the study conducted among older Taiwanese adults, reinforcing the importance of regularity in physical activity for positive mental health outcome as also demonstrated for other components like depression and sleep quality [5]. Likewise, in the experimental study using silver yoga exercise as an interventional measure, all the mental health indicators in the experimental group participants were found to be better than in the control group participants [22].

On the other hand, the experimental study, which utilized elastic band exercise as the intervention, found that self-perceived mental health status was not significantly different between the experimental and control groups either at the third month or at the end of interventional period of six months, compared to the baseline. However, it did find that the rate of decline in mental health score was greater in control group compared to the experimental group implying that the intervention did have a positive impact on the experimental group [19]. A significant positive association between physical activity and self-perceived mental health was also observed among senior adults in Czechia [34]. Similarly, Whitehall II cohort analysis among early old age adults from the UK also demonstrated a bidirectional association between physical activity and mental health. Physical activity and mental health improved over time and those with faster improvements in either variable experienced corresponding change in the other variable [35].
4.6. Limitations. The review attempted to include as many countries as possible from South Asia and Southeast Asia, but we could only review studies from 3 countries due to other studies not meeting the review eligibility criteria. Therefore, the findings might not apply to all countries from South and Southeast Asia. Likewise, all studies included in the review relied on subjective reporting of physical activity, which might not reflect the real levels of physical activity practiced by older adults. Due to limited countries included in the review, it was not possible to draw region-level comparative analyses. Likewise, the study only included the older adults without any diagnosed illnesses, so the study findings do not represent those older adults with one or more diagnosed illnesses. The study also has a limitation in terms of the time period covered by the review since only the studies conducted between 2008 and 2018 were included.

\section{Conclusion}

With the ever-increasing population of older people in South Asia and Southeast Asia, it is important to understand whether physical activity could be a way to improve the quality of life through positive mental health and wellbeing outcomes in older adults. As found in this review, factors like exercise frequency, regularity, and duration seem to have a positive impact on mental health and wellbeing of older adults. Likewise, any kind of intervention, which got older people to be more active, was found to positively contribute to their mental health and wellbeing as long as it was within a reasonable intensity. More wide-encompassing evidence through studies employing objectively measured physical activity incorporating various forms of physical activity/exercise practiced across different countries and cultures will help better inform public health strategies that can contribute to the positive mental health and wellbeing of older adults.

\section{Conflicts of Interest}

The authors declare that they have no conflicts of interest.

\section{Supplementary Materials}

Annex Figure 1: the figure is the spreadsheet matrix/format that was used to enter the information that was extracted from the articles listed for review. Annex Table 1: South Asian and South East Asian countries according to Asian Development Bank (ADB) and South Asian Association for Regional Cooperation (SAARC) classifications. Annex Table 2: Search grid example using the PubMed database. (Supplementary Materials)

\section{References}

[1] S. H. Chang, N. H. Chien, and M. C. Chen, "Regular Exercise and Depressive Symptoms in Community-Dwelling Elders in Northern Taiwan," Journal of Nursing Research, vol. 24, no. 4, p. 1, 2016. 
[2] World Health Organization, "Aeging and Health. Key facts," 2018. http://www.who.int/news-room/fact-sheets/detail/ageing-andhealth.

[3] World Health Organization, "Elderly population. Health situation and trend assessment 2018," 2018. http://www.searo. who.int/entity/health_situation_trends/data/chi/elderlypopulation/en/.

[4] World Health Organization, "Mental health of older adults. Factsheets 2017," 2017. http://www.who.int/news-room/factsheets/detail/mental-health-of-older-adults.

[5] C. Y. Wang, C.-J. Yeh, C.-W. Wang, C.-F. Wang, and Y.-L. Lin, “ The health benefits following regular ongoing exercise lifestyle in independent community-dwelling older Taiwanese adults," Australas Journal Ageing, vol. 30, no. 1, pp. 22-6, 2011.

[6] World Health Organization, "Key facts, Physical activity," 2018. http://www.who.int/en/news-room/fact-sheets/detail/physicalactivity.

[7] K. Makino, H. Ihira, A. Mizumoto, K. Shimizu, T. Ishida, and T. Furuna, "Associations between the settings of exercise habits and health-related outcomes in community-dwelling older adults," Journal of Physical Therapy Science, vol. 27, no. 7, pp. 2207-2211, 2015.

[8] Y. J. Lee and W. L. Hung, "The relationship between exercise participation and well-being of the retired elderly," Aging \& Mental Health, vol. 15, no. 7, pp. 873-881 2011.

[9] B. Guderian, A. Johnson, and V. Mathiowetz, "Impact of exercise frequency on hand strength of the elderly," Physical \& Occupational Therapy In Geriatrics, vol. 31, no. 3, pp. 268-279, 2013.

[10] N. Garatachea, O. Molinero, R. Martínez-García, R. JiménezJiménez, J. González-Gallego, and S. Márquez, "Feelings of well being in elderly people: relationship to physical activity and physical function," Archives of Gerontology and Geriatrics, vol. 48, no. 3, pp. 306-312 2009.

[11] A. Maramis, N. Van Tuan, and H. Minas, "Mental health in southeast Asia," The Lancet, vol. 377, no. 9767, pp. 700-702, 2011.

[12] S. David, R. Gazi, M. S. Mirzazada, C. Siriwardhana, S. Soofi, and N. Roy, "Conflict in South Asia and its impact on health," $B M J$, vol. 357, p. j1537, 2017.

[13] H. Arksey and L. O'Malley, "Scoping studies: towards a methodological framework," International Journal of Social Research Methodology, vol. 8, no. 1, pp. 19-32, 2005.

[14] A. C. Tricco, E. Lillie, W. Zarin et al., "Prisma extension for scoping reviews (PRISMA-ScR): checklist and explanation," Annals of Internal Medicine, vol. 169, no. 7, pp. 467-473, 2018.

[15] ADB, "Asian Development Bank. ADB Countries and Region.," https://www.adb.org/countries/main.

[16] SAARC, "South Asian Association for Regional Cooperation. SAARC Secretariat," http://www.saarc-sec.org/.

[17] M. A. Bhamani, M. M. Khan, M. S. Karim, and M. U. Mir, "Depression and its association with functional status and physical activity in the elderly in Karachi," Pakistan. Asian Journal of Psychiatry, vol. 14, pp. 46-51, 2015.

[18] P.-W. Ku and K. R. Fox, L.-J. Chen, "Physical activity and depressive symptoms in older adults: 11-year follow-up," Preventive Medicine, vol. 48, no. 3, pp. 250-255, 2012.

[19] S. Taboonpong and N. Puthsri, W. Kong-In, A. Saejew, “The effects of tai chi on sleep quality, well-being and physical performances among older adults. "' Pacific Rim International Journal of Nursing Research, vol. 12, no. 1, 2008.
[20] M.-C. Chen, H.-E. Liu, H.-Y. Huang, and A.-F. Chiou, “The effect of a simple traditional exercise programme (Baduanjin exercise) on sleep quality of older adults: a randomized controlled trial," International Journal Nursing Studies, vol. 49, no. 3, pp. 265-273, 2012.

[21] K.-M. Chen, M.-H. Chen, H.-C. Chao, H.-M. Hung, H.-S. Lin, and C.-H. Li, "Sleep quality, depression state, and health status of older adults after silver yoga exercises: cluster randomized trial," International Journal of Nursing Studies, vol. 46, no. 2, pp. 154-163, 2009.

[22] S.-Y. Chan and K. M. Chen, "Self-perceived health status and sleep quality of older adults living in community after elastic band exercises," Journal of Clinical Nursing, vol. 26, no. 13-14, pp. 2064-2072, 2017.

[23] P.-W. Ku, K. R. Fox, J. McKenna, and T.-L. Peng, "Prevalence of leisure-time physical activity in Taiwanese adults: results of four national surveys, 2000-2004," Preventive Medicine, vol. 43, no. 6, pp. 454-457, 2006.

[24] S. Joshi, J. Stephen, Gary J. Mooney et al., "Beyond METs: types of physical activity and depression among older adults," Age and Ageing, vol. 45, no. 1, pp. 103-109, 2016.

[25] P. Lampinen, R. L. Heikkinen, and I. Ruoppila, "Changes in intensity of physical exercise as predictors of depressive symptoms among older adults: an eight-year follow-up," Preventive Medicine, vol. 30, no. 5, pp. 371-380, 2000.

[26] K. Morgan and P. A. Bath, "Customary physical activity and psychological wellbeing: a longitudinal study," Age and Ageing, vol. 27, no. Suppl 3, pp. 35-40, 1998.

[27] V. Overdorf, B. Kollia, K. Makarec, C. A. Szeles, "The relationship between physical activity and depressive symptoms in healthy older women," Gerontology and Geriatric Medicine, vol. 2, 2016.

[28] M. Banno, Y. Harada, M. Taniguchi et al., "Exercise can improve sleep quality: a systematic review and meta-analysis," PeerJ, vol. 6, p. e5172, 2018.

[29] P.-Y. Yang, K.-H. Ho, H.-C. Chen, and M.-Y. Chien, "Exercise training improves sleep quality in middle-aged and older adults with sleep problems: a systematic review," Journal of Physiotherapy, vol. 58, no. 3, pp. 157-163, 2012.

[30] K. J. Reid, K. G. Baron, L. Brandon, E. Naylor, L. Wolfe, and P. C. Zee, "Aerobic exercise improves self-reported sleep and quality of life in older adults with insomnia," Sleep Medicine, vol. 11, no. 9, pp. 934-940, 2010.

[31] J. Li, B. Yang, M. Varrasse et al., "Physical activity in relation to sleep among community-dwelling older adults in China," Journal of Aging and Physical Activity, vol. 26, no. 4, pp. 647-654, 2018.

[32] Y. Netz, Wu Meng-Jia, Betsy Jane Becker, and Gershon Tenenbaum, "Physical activity and psychological well-being in advanced age: a meta-analysis of intervention studies," Psychology and Aging, vol. 20, no. 2, pp. 272-284, 2005.

[33] G. Windle, D. Hughes, P. Linck, I. Russell, and B. Woods, "Is exercise effective in promoting mental well-being in older age? A systematic review," Aging \& Mental Health, vol. 14, no. 6, pp. 652-669, 2010.

[34] J. Mudrák, P. Slepička, and I. Slepičková, "Perceived health and motivation to physical activity in seniors," Kontakt, vol. 16, no. 1, pp. e44-e50, 2014.

[35] S. Steinmo, G. Hagger-Johnson, and L. Shahab, "Bidirectional association between mental health and physical activity in older adults: Whitehall II prospective cohort study," Preventive Medicine, vol. 66, pp. 74-79, 2014. 


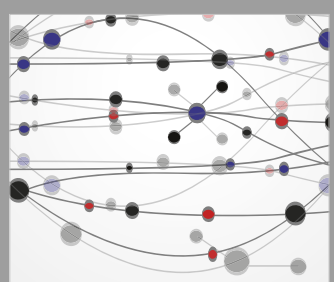

The Scientific World Journal
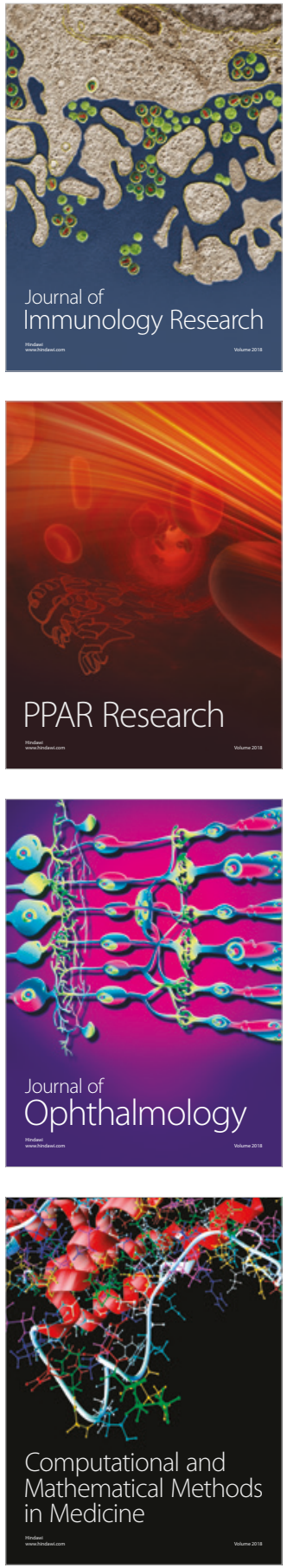

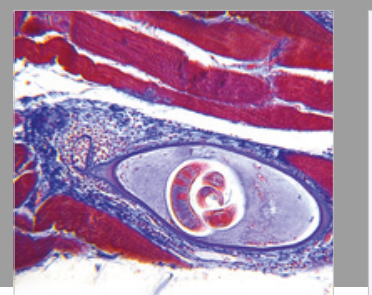

Gastroenterology Research and Practice

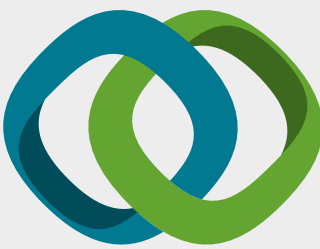

\section{Hindawi}

Submit your manuscripts at

www.hindawi.com
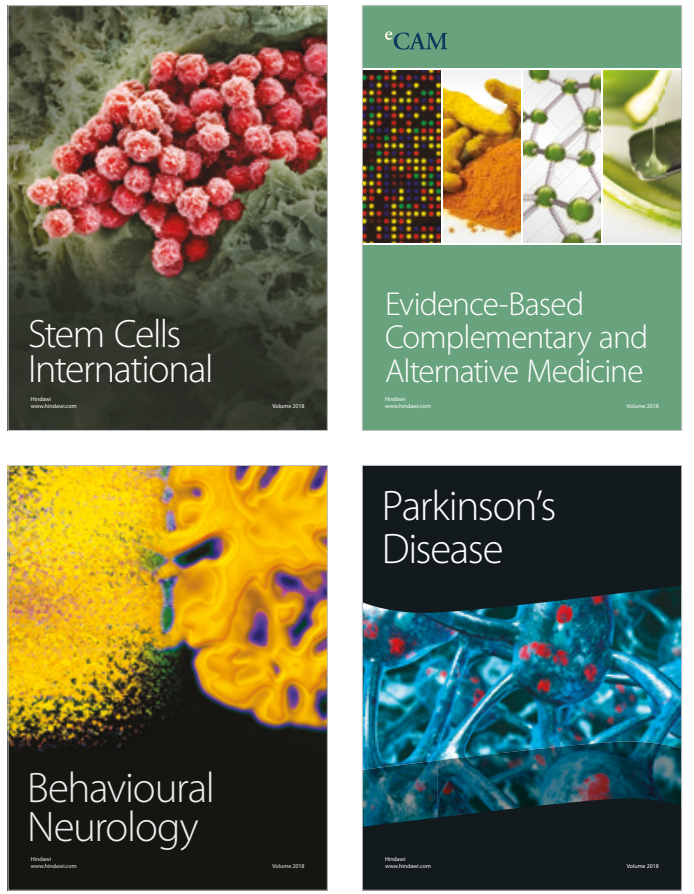

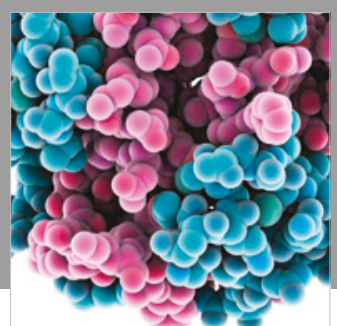

ournal of

Diabetes Research

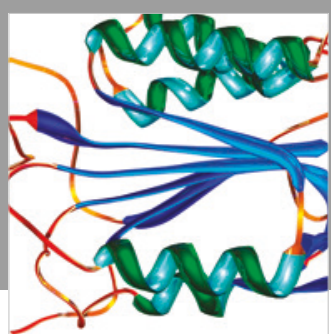

Disease Markers
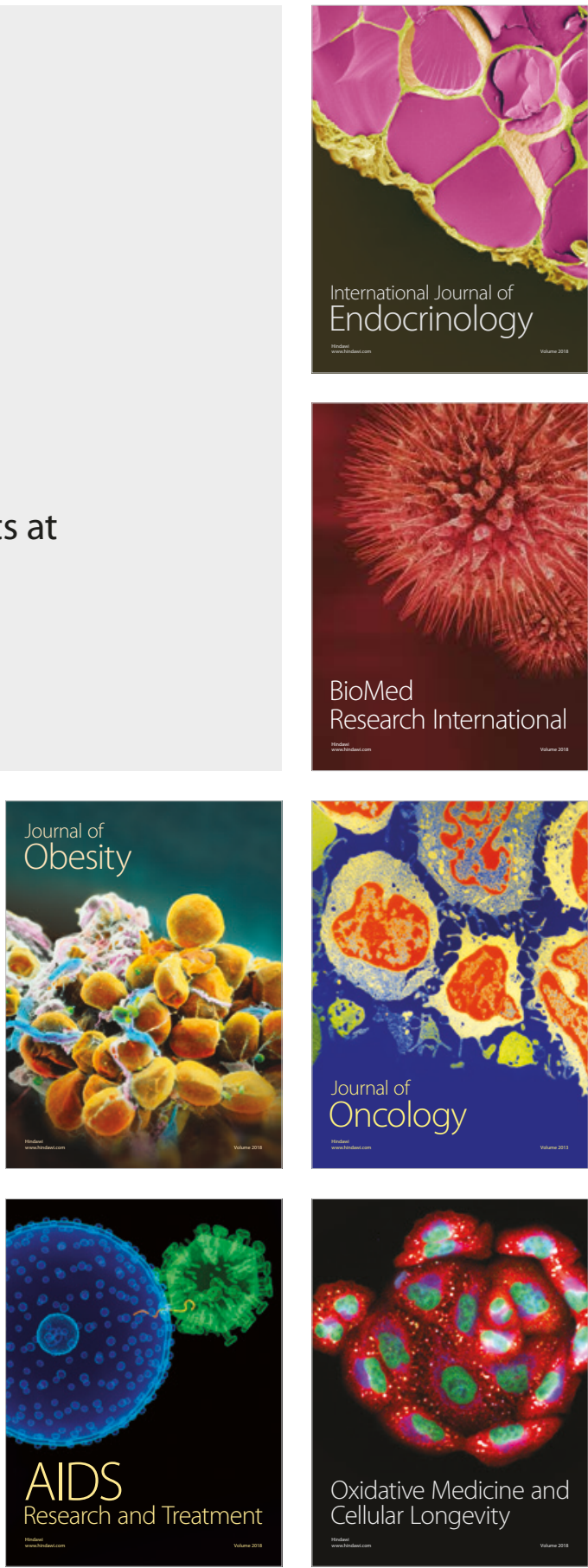